The Astrophysical Journal, 469:L135-L138, 1996 October 1

(c) 1996. The American Astronomical Society. All rights reserved. Printed in U.S.A.

\title{
CORONAL HEATING BY NANOFLARES AND THE VARIABILITY OF THE OCCURRENCE FREQUENCY IN SOLAR FLARES
}

\author{
Manolis K. Georgoulis and Loukas Vlahos \\ Section of Astrophysics, Astronomy, and Mechanics, Department of Physics, University of Thessaloniki, 54006 Thessaloniki, Greece \\ Received 1995 December 12; accepted 1996 July 10
}

\begin{abstract}
It has been proposed that flares in the solar corona may well be a result of an internal self-organized critical (SOC) process in active regions. We have developed a cellular automaton SOC model that simulates flaring activity extending over an active subflaring background. In the resulting frequency distributions we obtain two distinct power laws. That of the weaker events is shorter and much steeper (power law with index $\simeq-3.26$ ) than that of the intermediate and large events (power law with index $\simeq-1.73$ ). The flatter power law is in close agreement with observations of flares. Weaker events are responsible for $\simeq 90 \%$ of the total magnetic energy released, indicating a possible connection of nanoflares with coronal heating. Moreover, certain mechanisms cause the variability of the resulting indices and may provide answers to the problem of the variability of flares' occurrence frequency during the solar cycle.
\end{abstract}

Subject headings: stars: activity — stars: flares — Sun: activity — Sun: corona — Sun: flares —

Sun: magnetic fields

\section{INTRODUCTION}

Reconnecting current sheets (RCS) are considered to be the main energy release mechanism in solar and stellar active regions. Active regions are highly inhomogeneous, complex dynamical systems enclosing a large number of magnetic loops, randomly interacting in many independent places, and forming numerous current sheets with sizes varying over a wide range. Random photospheric motions shuffle and move the bases of the loops, adding complexity to the system. It seems that there is a critical threshold of marginal stability above which current sheets are canceled because of magnetic reconnection, in this way releasing energy in random places and making their neighborhood more probable to release energy as well. Depending on the sizes and the numbers of current sheets involved in an event, we may observe large energetic events (flares) and significantly weaker flaring activity (microflares). It has been proposed (Parker 1988) that even smaller energetic events, presently undetectable, extend below the observational limit of $10^{25}$ ergs. These extremely weak events are, in general, very short and have been named nanoflares.

Observations of flaring activity show that the peak-luminosity distribution of flares and microflares obeys a well-formed extended power law with an index $-1.80 \pm 0.05$ (Lin et al. 1984; Dennis 1985; Crosby, Aschwanden, \& Dennis 1992; Pearce, Rowe, \& Yeung 1993).

The rigidity of this index, however, is currently under serious consideration. Bai (1993), working on observational data, concluded that during the maximum activity of the 154 day periodicity the index is somewhat flatter, down to a limit of -1.68 , while during periods of low activity the spectrum steepens, showing an index of below -1.80 .

The energy released from flares provides a source of energy input to the corona. Ambient corona exhibits a temperature of about $2 \times 10^{6} \mathrm{~K}$. To maintain this temperature, chromospheric and coronal plasma need an energy supply of about $10^{7}$ ergs $\mathrm{cm}^{-2} \mathrm{~s}^{-1}$ (Withbroe \& Noyes 1977). Since active regions are driven by the convection zone-a highly turbulent unstable environment-it has been proposed that significant energy is supplied to the corona from a continuous, small-scale flickering of magnetic energy (Parker 1983; Van Ballegooijen 1986; Berger 1993). Parker (1989) proposed that nanoflares may heat chromosphere and corona.

However, if we extrapolate the observed frequency distributions to lower, presently unobservable energies, we conclude that the contribution of nanoflares to the total amount of energy released is insignificant. Hudson (1991) showed that if nanoflares show a power scaling with an index -4 or so, then they may be responsible for coronal heating. Porter, Fontenla, \& Simnett (1995) proved that if the index in the frequency distribution diagram is steeper than -2 for nanoflares, then nanoflares carry most of the energy released above an active region. Moreover, Litvinenko (1993) assumed that the probability function of an energetic event to occur is subject to a time-dependent transport equation, analogous to the usual distribution function for particles (Ginzburg \& Syrovatskii 1966). Based on this hypothesis, and adopting the theory of reconnecting current sheets (Imshennik \& Syrovatskii 1967), Litvinenko predicted theoretically a flaring index of about -1.75 , as well as a much steeper nanoflaring distribution below a cutoff energy limit. He also predicted that certain mechanisms, such as overlapping flares, may flatten the calculated flaring index.

Lu \& Hamilton (1991) were the first to apply self-organized criticality (SOC) in solar flares. The driver for the evolution of a magnetic topology is the magnetic gradient at a certain point. Lu \& Hamilton estimated the magnetic gradient at a certain site by averaging over the gradients (field differences) of the region over all its nearest six neighbors (we call this an "isotropic mode"). The results of their simulation showed that the peak-luminosity (or total-energy) index had a unique value, approximately equal to -1.8 . This result contradicts the observational results of Bai (1993) and the theoretical considerations of Litvinenko (1993).

We have developed an isotropic SOC model in which an 
explosion affects its neighborhood by lowering the instability criteria and igniting secondary bursts triggered by an initial instability in an avalanche-type manner (Georgoulis, Kluiving, $\&$ Vlahos 1995). The model appears to strengthen significantly the SOC behavior, also leading to close agreement with observations of flares (peak-luminosity index close to -1.8 ). In an attempt to investigate the role of anisotropy (we do not average over the six gradients but we deal with each one of them independently) on the system, we have developed an "anisotropic model" in which only numerous weak events appear. The peak-luminosity frequency distribution is still a power law but it is much shorter and considerably steeper, with an index around -3.6 (Vlahos et al. 1995).

In this Letter, we introduce a new cellular automaton model in which we attempt a merging of the two independent physical processes (isotropic and anisotropic). The rules of the model decide the way an instability will be relaxed (isotropically or anisotropically). Furthermore, the introduction of a variable loading mechanism causes the variability of the resulting power-law indices. A description of the model is presented in $\S 2$. The results of our model are outlined in $\S 3$, while in $\S 4$ we discuss the possible physical interpretation of these results.

\section{THE MODEL}

Our model is a "Three-Dimensional Sandpile Model." The basic rules of the algorithm are given below:

1. Initial loading.-A cubical grid is constructed, and with each grid point a scalar "field" is associated. The grid attempts to simulate a limited part of the upper photosphere, together with the chromospheric and coronal layers directly above. The scalar quantities represent the local values of the magnetic field at various locations. The starting configuration is random and corresponds to a quiescent initial situation.

2. Loading.-Randomly selected lattice sites gain scalar increments with a rate equal to one point per iteration. In all previous studies (Lu \& Hamilton 1991; Vlahos et al. 1995; Georgoulis et al. 1995), the increment used was constant and small. We now use a variable loading mechanism for which the probability $P(\delta B)$ for a certain increment $\delta B$ to occur is

$$
P(\delta B)=A(\delta B)^{-\alpha},
$$

where $A$ and $\alpha$ are constants. Using equation (1), one can obtain values of $\delta B$ from practically zero up to very large numbers. Constant alpha $(A)$ prevents $\delta B$ to obtain unacceptably large values. Scalars $\delta B$ correspond to the values of magnetic fields injected to the chromosphere and corona from the convection zone.

If a randomly selected grid point gains an increment $\delta B$ according to equation (1), its first- and second-order neighbors gain a small portion of $\delta B$, arbitrarily set to $25 \%$ and $10 \%$, respectively.

3. Criteria for instabilities.-Possible instabilities are triggered with respect to "field gradients," i.e., field differences between a grid point and its vicinity. Using arbitrary units at this stage, we introduce a certain critical threshold (i.e., a critical "gradient steepness") $B_{\text {cr }}$, above which a topology should be considered as unstable. What is to be compared to that threshold is the "slope" at a certain grid point. The slope at a grid point $i$ can be either the field difference $S_{\mathrm{i}}$ between that site and the average of its first-order vicinity (isotropic case)

$$
S_{i}=B_{i}-\frac{1}{6} \sum_{j} B_{i j},
$$

or the field differences $S_{i j}$ between that site and each one of its first-order neighbors separately (anisotropic case),

$$
S_{i j}=B_{i}-B_{i j},
$$

where $B_{i}$ is the value of the local magnetic field at the location $i$ and $B_{i j}(j=1,6$ for every $i)$ are the magnetic field values of

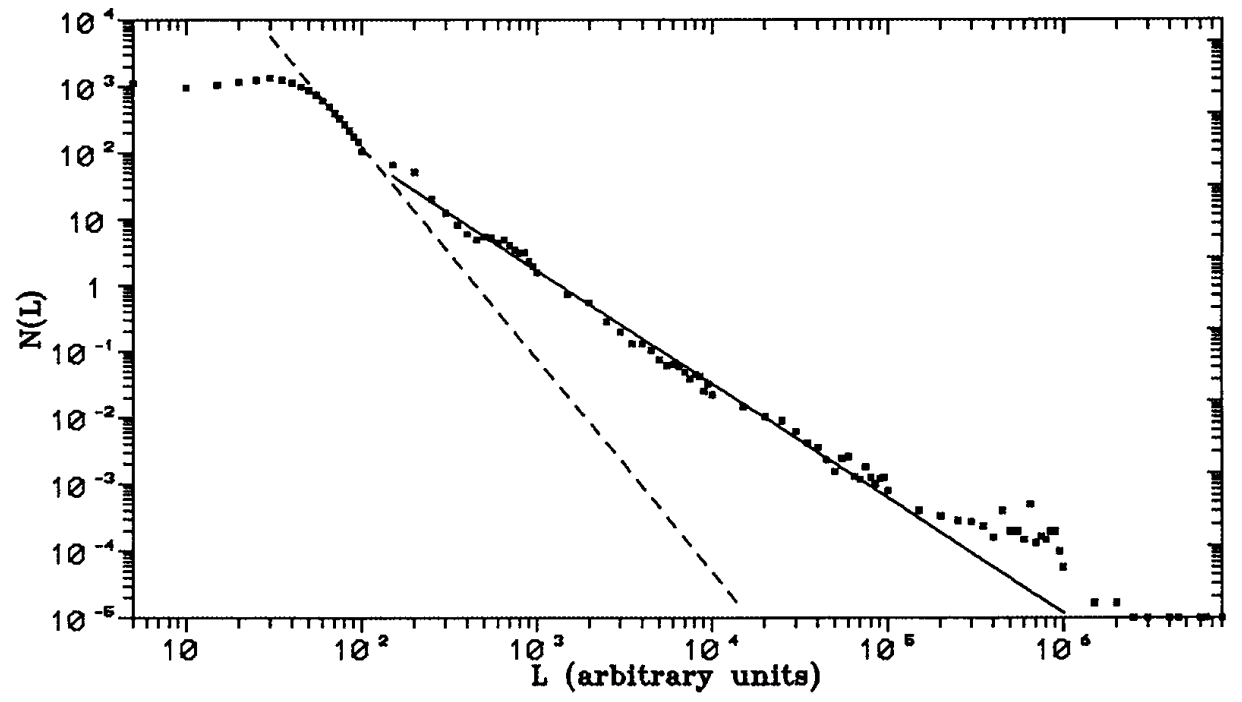

FIG. 1. - Typical peak-luminosity frequency distribution for a $150 \times 150 \times 150$ grid. Distribution is the average of 10 sample runs. 

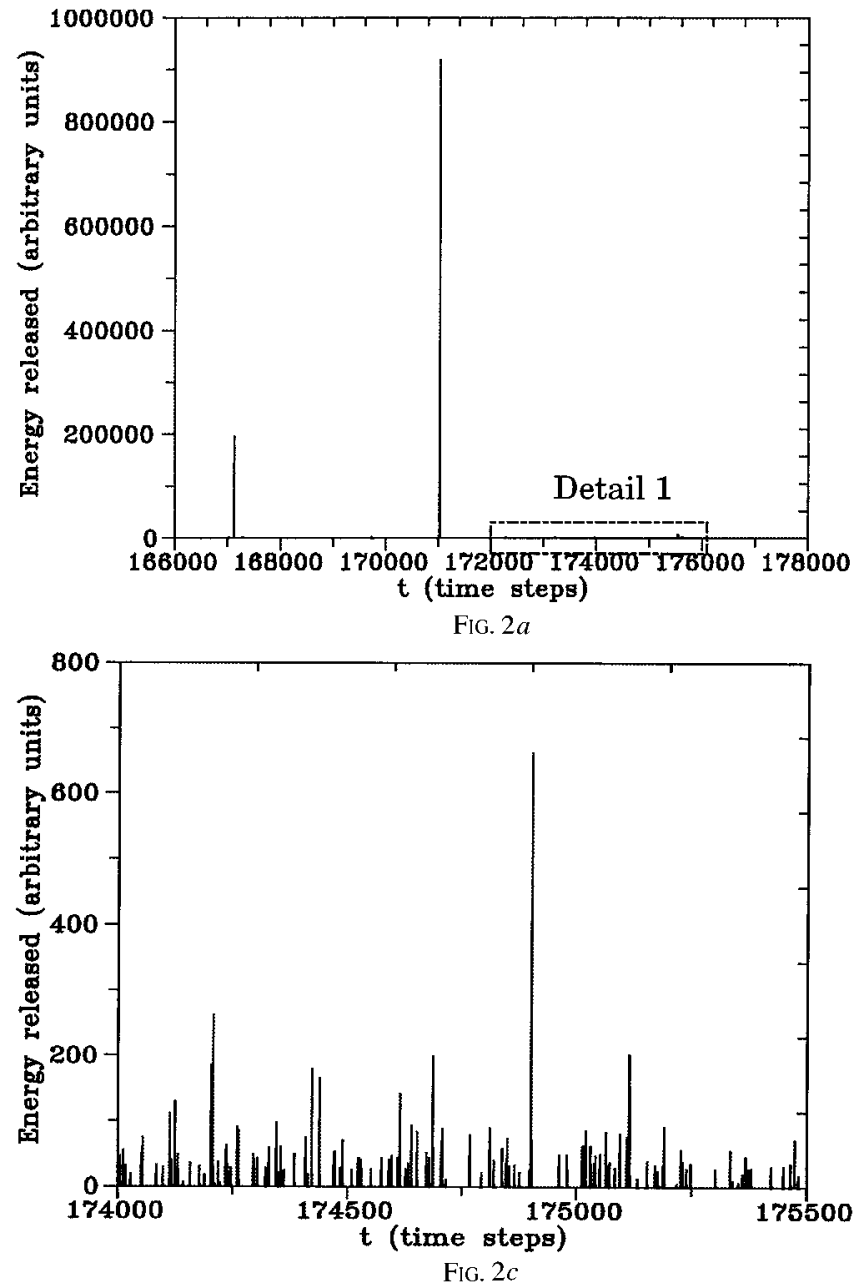

FIG. 2.-Time series of the energetic events obtained: $(a)$ unscaled part of the time series; $(b)$ rescaled "Detail 1;" (c) rescaled "Detail 2."

its six first-order neighbors. The instability criterion is defined by the relations

$$
S_{i}>B_{\text {cr }} \quad \text { or } \quad S_{i j}>B_{\text {cr }} .
$$

If either of the inequalities (4) is satisfied, the topology is considered unstable. Relaxation of gradients proceeds (1) by redistributing the excess field to the vicinity of the unstable site, and (2) by releasing energy.

4. Field Relaxation. - If the slope $S_{i}$ exceeds the critical threshold $B_{\text {cr }}$ because of equation (4), then automaton rules for field restructuring are stated as

$$
\begin{gathered}
B_{i} \rightarrow B_{i}-\frac{6}{7} B_{\text {cr }} \\
B_{i j} \rightarrow B_{i j}+\frac{1}{7} B_{\mathrm{cr}}, \quad j=1,6 .
\end{gathered}
$$

Similarly, if one (or more) slope(s), $S_{i j}$, satisfies equation (4), then the field redistribution is governed by the relations

$$
\begin{gathered}
B_{i} \rightarrow B_{i}-\frac{6}{7} B_{\mathrm{cr}} \\
B_{i j} \rightarrow B_{i j}+\delta_{j},
\end{gathered}
$$

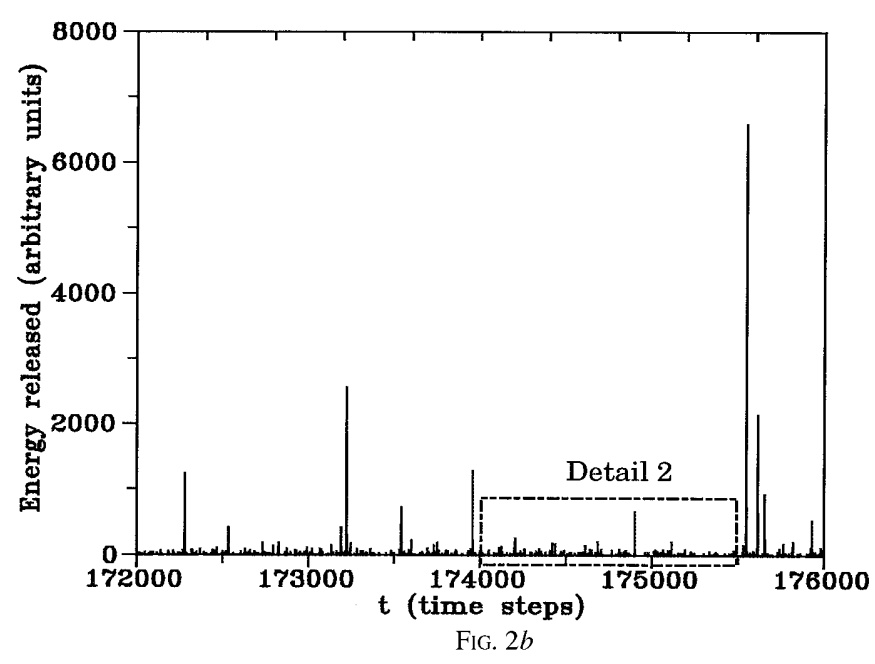

provided that

$$
\sum \delta_{j}=\frac{6}{7} B_{\text {cr }} \quad \text { and } \quad \delta_{j}=\frac{6}{7} B_{\text {cr }} \frac{S_{i j}}{\sum_{j} S_{i j}}, j=1,6 .
$$

The sum in the denominator of equation (9) stands only for those slopes, $S_{i j}$, that satisfy equation (4). In the case of equations (5) and (6), the restructuring of the topology affects equally the entire first-order neighborhood of the unstable point $i$, while in the case of equations (7), (8), and (9) relaxation provides a directionality toward the first-order neighbor(s) that exhibit an abrupt field difference with respect to the point of consideration. The rest of the neighborhood remains unaffected.

A cascade of events may be triggered by a seed instability caused by the mechanism described above. Furthermore, the model predicts the ignition of secondary bursts (i.e., bursts that occur when the gradients do not exceed the critical threshold). For a detailed description, see Vlahos et al. (1995) and Georgoulis et al. (1995).

5. Energy release.-In both cases (isotropic or anisotropic), the energy released during field restructuring is equal to a quantity $\left(B_{i}-6 B_{\mathrm{cr}} / 7\right)^{2}$, which is to be associated with magnetic energy release during magnetic reconnection.

If a certain $\delta B$ triggers an instability, either by equation (2) or equation (3), then we stop dropping increments until the instability has completely relaxed. We scan and rescan the grid to accomplish this. Each scanning corresponds to one time step. Thus, an iteration may consist of more than one time steps. The energy released during an iteration is treated as the total energy of the event, while peak luminosity is defined as the energy released per time step. Thus, total energy, peak luminosity, and duration of an event are all properly quantified.

\section{RESULTS}

A typical peak-luminosity frequency distribution is illustrated in Figure 1. It is clear that the distribution consists of two different components. Intermediate and higher energies obey a well-defined power law with an index $\delta_{1}=-1.73 \pm 0.05$ (solid line), extending over 3 orders of magnitude or so. Low-energy band, however, exhibits a much steeper, short power law with an index $\delta_{2}=-3.26 \pm 0.2$ (dashed line), ex- 
tending over much less than an order of magnitude. Power laws are also obtained for total-energy and duration distributions. Indices are insensitive to the selection of the critical threshold $B_{\text {cr }}$. Note that the index of the flatter power law is closely consistent with observations (Lin et al. 1984; Dennis 1985). We should emphasise that indices $\delta_{1}$ and $\delta_{2}$ are not unique; they are closely related to the value of index $\alpha$ in equation (1). Lower values of $\alpha$ cause a flattening of both power laws of the distribution, while higher values cause a steepening of both indices. The values of $\delta_{1}, \delta_{2}$ are also dependent on the number of points per iteration that will be randomly selected to receive the increment $\delta B$. A complete study on the variability of the occurrence frequency indices will follow in a forthcoming publication.

The transition of the behavior between higher and lower energies can be clearly seen in the logarithmic distribution of Figure 1. In the time series of the events recorded, however, the weaker events that form the steeper part of the distribution are practically undetectable. To verify this, we have plotted a part of the unscaled time series in Figure $2 a$. The dashed box "Detail 1" is rescaled and plotted in Figure $2 b$, clearly demonstrating a hidden background of events extending orders of magnitude below the well-formed large bursts. The dashed box "Detail 2" of Figure $2 b$ is further rescaled and plotted in Figure $2 c$. Now the background of numerous, extremely weak events is clearly exposed, showing that the time series obtained from the model enclose a remarkable microstructure composed by swarms of short and spiky energetic events.

The most promising result, however, is that the sharp part of the frequency distribution is actually responsible for the vast majority of energy release. Integrating the total-energy frequency distribution, we found that the low-energy band contributes $91.2 \% \pm 0.3 \%$ of the total energy released. Intermediate and large events contribute the remaining $8.8 \%$. Flattening or steepening of the indices $\delta_{1}, \delta_{2}$ for $\delta_{1}$ within the limits of observations does not seriously affect the rigidity of this result, adding a fluctuation of less than 5\%. As a conse- quence, the large energetic events, although quite impressive, are statistically insignificant for the energy release process.

\section{SUMMARY AND CONCLUSIONS}

The results presented above indicate that the observations of flares and microflares and their statistical properties can be reproduced if active regions are treated as an SOC dynamical system. Moreover, a single loading method (eq. [1]) can give rise to the emergence of two distinct power laws, one dominating the weaker energetic events and the other providing the scaling behavior of intermediate and large events. We associate the weakest events recorded with nanoflares, and the rest with microflares and flares, respectively.

The indices of the two different parts of the frequency distribution are not unique; they seem to depend on (1) how intensive the loading procedure is (the value of exponent $\alpha$ in eq. [1]), and (2) the probability of overlapping events (the number of points randomly chosen to receive the injected field in one iteration). Thus, we may have some preliminary indications concerning the nature of the variability of flares' occurrence frequency.

Our results support the theoretical considerations of Litvinenko (1993). Nanoflares seem to form a steeper power law. Furthermore, either nanoflares or microflares and flares are all consequences of a threshold-nature instability.

Finally, our results support the hypothesis of coronal heating by nanoflares (Parker 1989). We believe that the still undetected part of the flaring activity plays a crucial role in the energy release process and may be responsible for heating the corona. Improved observational facilities will probably add the last word on the existence of nanoflares and their potential role for coronal heating.

We are grateful to Rene Kluiving for a fruitful cooperation that greatly helped the completion this letter. We also thank N. K. Spyrou and I. Seiradakis for their comments in the text.

\section{REFERENCES}

Bai, T. 1993, ApJ, 404, 805

Berger, M. A. 1993, Phys. Rev. Lett., 70, 705

Crosby, N. B., Aschwanden, M. J., \& Dennis, B. R. 1992, Sol. Phys. 143, 257

Dennis, B. R. 1985, Sol. Phys., 100, 465

Georgoulis, M., Kluiving, R., \& Vlahos, L. 1995, Physica A, 218, 191

Ginzburg, V. L., \& Syrovatskii, S. I. 1966, Origin of Cosmic Rays (New York: Macmillan)

Hudson, H. S. 1991, Sol. Phys., 133, 357

Imshennik, V. S., \& Syrovatskii, S. I. 1967, Soviet Phys.-JETP, 25, 656

Lin, R. P., Schwartz, R. A., Kane, S. R., Pelling, R. N., \& Hurly, C. C. 1984, ApJ, 285, 421
Litvinenko, Y. E. 1993, Sol. Phys., 151, 195

Lu, E. T., \& Hamilton, R. J. 1991, ApJ, 380, L89

Parker, E. N. 1983, ApJ, 264, 642

1988, ApJ, 330, 474

Pearce, J., Rowe, A. K., \& Yeung, J. 1993, Ap\&SS, 208, 99

Porter, J. G., Fontenla, J. M., \& Simnett, G. M. 1995, ApJ, 438, 472

Van Ballegooijen, A. A. 1986, ApJ, 311, 1001

Vlahos, L., Georgoulis, M., Kluiving, R., \& Paschos, P. 1995, A\&A, 299, 897

Withbroe, G. L., \& Noyes, R. W. 1977, ARA\&A, 15, 363 